\title{
Further Differences in Biochemical Composition of Roots of Ni-Hyperaccumulating and Non-Hyperaccumulating Genotypes of Senecio coronatus
}

\author{
J. Mesjasz-Przybylowicz ${ }^{1}$, A.D. Barnabas ${ }^{1}$ I. Yousef $^{2}$, P. Dumas², F. Jamme², T.P. Sechogela¹, W.J. \\ Przybylowicz ${ }^{1,3}$ \\ 1. iThemba LABS, National Research Foundation, PO Box 722, 7129 Somerset West, South Africa \\ 2. Soleil Synchrotron, BP 48 F91192 Gif sur Yvette Cedex, France \\ 3. AGH University of Science and Technology, Faculty of Physics \& Applied Computer Science, al. A. \\ Mickiewicza 30, 30-059 Krakow, Poland
}

Hyperaccumulation is an unusual plant response to metaliferous soils. Such soils like those derived from ultramafic rocks, have elevated concentrations of heavy metals, mainly Ni, Cr, Zn and Cd. Most plants growing on these soils exclude metals from their shoots as excessive accumulation of heavy metals is toxic to the majority of plants. However, about $2 \%$ of plants growing on metaliferous soils take up and accumulate large quantities of metals in their shoots: a phenomenon known as hyperaccumulation. Senecio coronatus (Thunb) Harv, a widespread South African plant, also occurs on ultramafic outcrops where two genotypes have been identified: a Ni-Hyperaccumulator $(\mathrm{H})$ and a Non-Hyperaccumulator (NH).In previous studies [1,2] some aspects of differences in cytology and chemical composition of the roots of the genotypes were reported. The present study details further biochemical differences between the roots and the possible relationship of these to the differential uptake of $\mathrm{Ni}$.

Chemical composition information was obtained using high spatial resolution synchrotron infrared microspectroscopy on thin vibratomed root sections $50 \mathrm{~mm}$ from the root tips, at the SOLEIL Synchrotron Facility in France (SMIS beam line).

As described in a previous study [1], distinct groups of cells were present in the inner cortex of the $\mathrm{H}$ on the same radius as the xylem and phloem elements (Fig.1A, arrow) but the equivalent cells in the same region of the cortex of the $\mathrm{NH}$ were not as distinct (Fig.1E, arrow). To obtain more information about these cell groups, their biochemical composition was investigated. Spectra were recorded within the rectangular areas demarcated by red lines in the optical images of the $\mathrm{H}$ (Fig.1B) and the $\mathrm{NH}$ (Fig.1F). Comparison of the average normalized spectra of the $\mathrm{H}$ (Fig.1HS, blue spectrum) and the $\mathrm{NH}$ (Fig.1HS, red spectrum) showed that a significant difference between them was in the C-H stretching region (3100-2800 $\mathrm{cm}^{-1}$ ) often attributed to lipids. The strongest absorbance bands in the $\mathrm{H}$ appeared at 2920 and $2850 \mathrm{~cm}^{-1}$ which can be assigned to asymmetrical and symmetrical C-H stretching vibrations respectively. A major difference therefore between the roots was the increased lipid concentration in the $\mathrm{H}$. Chemical profiles confined to the rectangular areas of the optical images of the genotypes (Fig.1B,F) were also constructed using the integrated absorbance of the $\mathrm{CH}$ stretching region between $3100-2800 \mathrm{~cm}^{-1}$. Comparison of these chemical profiles clearly show many more strong absorbance areas in the cell group of the $\mathrm{H}$ (Fig.1C) compared to that of the NH (Fig.1 G) confirming the higher concentration of lipids in the former.

Another difference between spectra of the genotypes was the presence of a small band at $3010 \mathrm{~cm}^{-1}$ in the $\mathrm{C}-\mathrm{H}$ stretching region of the $\mathrm{H}$ (Fig.1HS). This band is attributed to $\mathrm{H}-\mathrm{C}=\mathrm{C}$ unsaturated $\mathrm{C}-\mathrm{H}$ stretching, possibly from unsaturated lipids. Chemical imaging of this band in the frequency region 3030-2990 $\mathrm{cm}^{-1}$ showed that these unsaturated vibrational bands were superimposed on the lipid 
localization sites of the H (Fig.1D).

A third difference between the spectra of the genotypes was the detection of a slight shoulder at 1465 $\mathrm{cm}^{-1}$ in the spectrum of the $\mathrm{H}$ (Fig.1HS) assigned to CH2 bending, probably from lipids [3].No differences were found in spectra of the polysaccharide region between 1200-800 $\mathrm{cm}^{-1}$. Principal component analysis (PCA) on the full range $(4000-2800+1800-800)$ as well as PCA loadings plots of the PC1 component were also carried out and these confirmed the spectral differences observed in the genotypes. The significance of increased lipid levels and possibly also of different lipid types in roots of the $\mathrm{H}$, compared to those of the $\mathrm{NH}$, is presently not clear but may be a reflection of stress caused by the presence of higher Ni concentrations in the roots of the former.

[1] Mesjasz-Przybylowicz J, Barnabas A, Przybylowicz W, Plant Soil 293(2007):61-78.

[2] Mesjasz-Przybylowicz J, et al, Microscopy and Microanalysis 20(2014):1276-1277.

[3] Komal Kumar J, Devi Prasad A G, Romanian Journal of Biophysics 21 (2011):63-71.

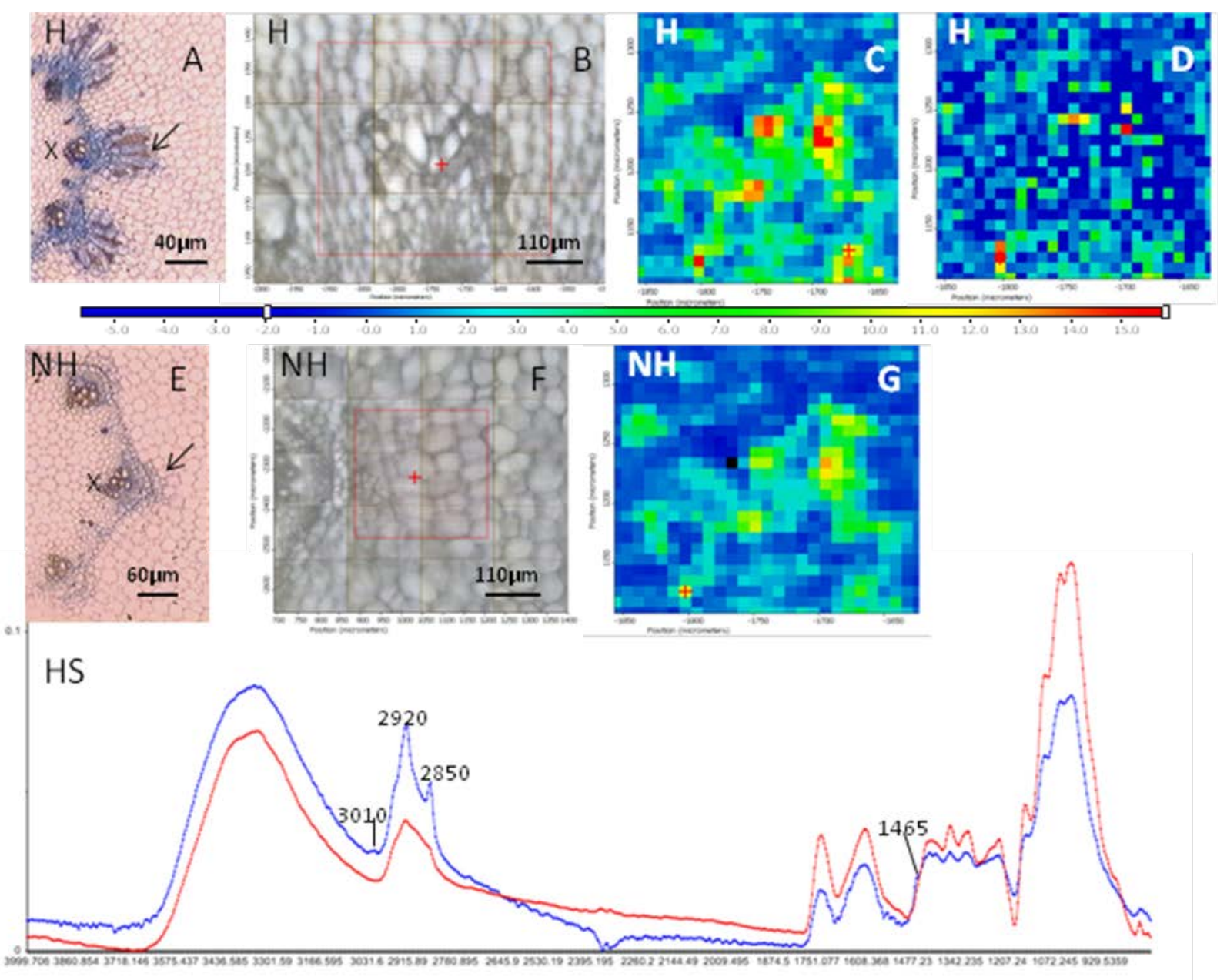

Fig.1 A-HS: Optical images (A,B,E,F) and chemical profiles (C,D,G) of inner cortical cell groups in roots of Senecio coronatus genotypes.The warmer colours in C,D and G (towards the red end of the colour scale) indicate higher absorbance. Average normalized spectra (HS) of Ni- Hyperaccumulator (blue spectrum) and Non-Hyperaccumulator (red spectrum). H, Ni-Hyperaccumulator; NH, NonHyperaccumulator; X, xylem. 